\title{
Doença hepática gordurosa não alcoólica: evolução e risco de desenvolvimento de cirrose hepatica
}

\author{
Non-alcoholic fatty liver disease: progression and risk of liver cirrhosis development \\ Enfermedad del hígado graso no alcohólico: evolución y riesgo de desarrollar cirrosis \\ hepática
}

Beatriz Cunta Gonçalves ${ }^{1 *}$, Carla Cristina de Freitas Rosa ${ }^{2}$, Cecília Soares Tôrres ${ }^{3}$, Joyce Vilarins Santos Soares ${ }^{4}$, Karolayne Verdan de Alencar Costa ${ }^{5}$, Leticia Stefanelli Potsch ${ }^{6}$, Lorena de Almeida Ludovino ${ }^{7}$, Sophia Bark ${ }^{4}$, Vinícius Barbosa dos Santos Sales ${ }^{8}$, Renan Guimarães Assunção Campos ${ }^{9}$.

\section{RESUMO}

Objetivo: Relacionar a Doença Hepática Gordurosa não Alcoólica (DHGNA) e o risco de cirrose, além de renovar e descrever os conhecimentos científicos sobre o tema nos últimos cinco anos. Revisão bibliográfica: A DHGNA é definida pelo acúmulo anormal de gordura nos hepatócitos. É considerado um problema de saúde pública, em razão da sua alta prevalência e a possível progressão para cirrose e/ou carcinoma hepatocelular. Os fatores de risco são diferenciados em modificáveis e não modificáveis, tendo a obesidade como enfermidade de base para o desenvolvimento da DHGNA. Apresentam variadas fases de progressão, as quais incluem esteatose hepática, Esteato-Hepatite Não Alcoólica (EHNA), fibrose hepática, cirrose hepática e carcinoma hepatocelular. A DHGNA apresenta associações com outras comorbidades como síndrome metabólica, diabetes mellitus tipo 2 (DMT2) e dislipidemia aterogênica. Considerações finais: A DHGNA consiste no acúmulo de gordura nos hepatócitos de indivíduos que não consomem ou consomem menos que $30 \mathrm{~g}$ de álcool por dia propiciando um distúrbio metabólico, de evolução progressiva e geralmente assintomática, resultando em diagnósticos tardios e aumentando o risco de evoluir para cirrose e/ou carcinoma hepatocelular.

Palavras-chave: Adultos, Cirrose hepática, Hepatopatia gordurosa não alcoólica.

\begin{abstract}
Objective: To relate non-alcoholic fatty liver disease (NGAHD) and the risk of cirrhosis, and to renew and describe the scientific knowledge on the subject over the past five years. Bibliographic review: NASH is defined by the abnormal accumulation of fat in hepatocytes. It is considered a public health problem due to its high prevalence and possible progression to cirrhosis and/or hepatocellular carcinoma. Risk factors are differentiated into modifiable and non-modifiable, with obesity as the underlying disease for the development of $\mathrm{NASH}$. They present various stages of progression, which include hepatic steatosis, Non-Alcoholic Steatohepatitis (NHEA), hepatic fibrosis, cirrhosis and hepatocellular carcinoma. NASHD presents associations with other comorbidities such as metabolic syndrome, type 2 diabetes mellitus (DMT2) and atherogenic dyslipidemia. Final considerations: NASH consists of the accumulation of fat in the hepatocytes of individuals who do not consume or consume less than $30 \mathrm{~g}$ of alcohol per day, leading to a metabolic disorder, progressive and generally asymptomatic, resulting in late diagnosis and increasing the risk of developing cirrhosis and/or hepatocellular carcinoma.
\end{abstract}

Keywords: Adult, Liver cirrhosis, Non-alcoholic fatty liver disease.

1 Universidade do Estado do Rio de Janeiro (UERJ), Rio de Janeiro - RJ.

*E-mail: goncalves.beatriz@graduacao.uerj.br

2 Universidade de Franca (UNIFRAN), Franca - SP.

${ }^{3}$ Centro Universitário UniFacid, Teresina - PI.

${ }^{4}$ Instituto Tocantinense Presidente Antônio Carlos (ITPAC), Palmas - TO.

${ }^{5}$ Universidad Cristiana da Bolívia (UCEBOL), Santa Cruz de la Sierra, Bolívia.

6 Universidade Vila Velha (UVV), Vila Velha - ES.

7 Faculdade Santo Agostinho (FASAI), Itabuna - BA.

8 Universidade Federal de Sergipe (UFS), Lagarto - SE.

${ }^{9}$ Centro Universitário de Patos de Minas (UNIPAM), Patos de Minas - MG.

SUBMETIDO EM: 3/2021

ACEITO EM: 4/2021

PUBLICADO EM: 5/2021 


\section{RESUMEN}

Objetivo: Relacionar la enfermedad del hígado graso no alcohólico (HGNA) y el riesgo de cirrosis, y renovar y describir los conocimientos científicos sobre el tema en los últimos cinco años. Revisión bibliográfica: La EHNA se define por la acumulación anormal de grasa en los hepatocitos. Se considera un problema de salud pública debido a su alta prevalencia y a la posible progresión a cirrosis y/o carcinoma hepatocelular. Los factores de riesgo se diferencian en modificables y no modificables, siendo la obesidad la enfermedad subyacente para el desarrollo de la EHNA. Presentan varios estadios de progresión, que incluyen la esteatosis hepática, la Esteatohepatitis no Alcohólica (ESA), la fibrosis hepática, la cirrosis hepática y el carcinoma hepatocelular. La NASHD presenta asociaciones con otras comorbilidades como el síndrome metabólico, la diabetes mellitus de tipo 2 (DMT2) y la dislipidemia aterogénica. Consideraciones finales: La EHNA consiste en la acumulación de grasa en los hepatocitos de individuos que no consumen o consumen menos de $30 \mathrm{~g}$ de alcohol al día, dando lugar a un trastorno metabólico, progresivo y generalmente asintomático, que da lugar a un diagnóstico tardío y aumenta el riesgo de desarrollar cirrosis y/o carcinoma hepatocelular.

Palabras clave: Adulto, Cirrosis hepática, Enfermedad del hígado graso no alcohólico.

\section{INTRODUÇÃO}

A Doença Hepática Gordurosa não Alcoólica (DHGNA) é definida pela concentração de gordura nos hepatócitos, podendo acarretar no aumento da massa hepática em até $10 \%$ do peso em indivíduos que não consomem ou consomem baixa quantidade de álcool. Ela apresenta diferentes graus de alterações histológicas, sendo elas a esteatose hepática simples, a Esteato-Hepatite Não Alcoólica (EHNA) com presença de inflamação, balonização dos hepatócitos, dano hepático e fibrose em graus variados -, podendo evoluir para cirrose hepática e carcinoma hepatocelular (OTT-FONTES PR, et al., 2020).

Estima-se que a DHGNA afete, em média, $5 \%$ da população dos Estados Unidos, tratando-se da segunda maior causa de transplante hepático. É caracterizada pela presença de esteatose hepática, inflamação e vários graus de fibrose hepática. Pacientes que desenvolvem a fibrose hepática avançada possuem maior risco de infecção hepática e carcinomas (LOOMBA R, et al., 2018). O aumento no teor de gordura do fígado na DHGNA não é explicado pelo consumo de álcool e hoje é considerado a principal causa de doença hepática (PELUSI, et al., 2019). A DHGNA é encarada como o transtorno hepático mais comum no mundo, com prevalência de até $35 \%$ (OTT-FONTES PR, et al., 2020).

A DHGNA é considerada uma doença progressiva e assintomática, comumente diagnosticada mais tardiamente, em uma fase na qual as taxas de mortalidade são maiores e a intervenção já não é tão eficaz (JARVIS H, et al, 2020). Histologicamente, ela é predominantemente caracterizada por esteatose macrovesicular, e é reconhecida quando o acúmulo de gordura está associado um ou mais dos seguintes achados: inflamação, balonização dos hepatócitos e fibrose (ANDRADE GC, et al., 2016).

A DHGNA está comumente associada à obesidade, à dislipidemia e à resistência à insulina (RI), que são fatores da síndrome metabólica. Tal comorbidade pode ser desencadeada em razão de hábitos alimentares inadequados e do sedentarismo, condições que levam ao excesso de peso e desencadeiam em doenças associadas (OTT-FONTES PR, et al., 2020).

Um ponto crucial no tratamento da DHGNA é a diferenciação de esteato-hepatite alcoólica e a esteatose simples, assim como o estadiamento da fibrose hepática, uma vez que os pacientes com esteato-hepatite não alcoólica possuem mais risco de desenvolver complicações como cirrose e carcinoma hepatocelular (CASTERA L, 2018).

Atualmente a obesidade vem crescendo de forma alarmante em todo o mundo, concomitantemente aumentam os casos de DHGNA. A prevalência de DHGNA em pacientes obesos consiste em cerca de $30 \%$ a 37\%, e aqueles com altos índices de gordura visceral e circunferência abdominal aumentada estão em maior risco de desenvolver tal enfermidade. $O$ achado mais comum dentre esses indivíduos trata-se da esteatose hepática simples sem fibrose significativa, o grau mais leve de DHGNA (PERUMPAIL BJ, et al., 2017).

Portanto, o presente estudo teve por objetivo realizar uma revisão de literatura sobre a relação da DHGNA e o risco de cirrose, assim como renovar e descrever os conhecimentos científicos sobre o tema nos últimos cinco anos. 


\section{REVISÃO BIBLIOGRÁFICA}

\section{Definição e Fisiopatologia}

As doenças do fígado estão alicerçadas em 3 lesões principais, as quais representam o aumento do nível de gravidade da patologia, seja por vias alcoólicas ou não. Tais fases apresentam-se como esteatose, hepatite e cirrose (falência do órgão). É importante salientar que o gerenciamento de risco das doenças hepáticas deve ser prioridade em qualquer conduta médica, haja vista que o objetivo consiste em impedir a progressão da lesão para cirrose e/ou hepatocarcinoma (PARENTE DB, 2020). No presente estudo, a DHGNA será o destaque.

A DHGNA é definida pelo acúmulo anormal de gordura nos hepatócitos por meio da deposição de triglicerídeos, ácidos graxos, monoglicerídeos e diglicerídeos, pertencentes à forma histológica macrovesicular, a mais frequente, com prevalência na parte centro lobular do fígado (zonas 2 e 3). No entanto, pode ser difusa em casos mais avançados. A doença gordurosa não alcoólica é considerada um problema de saúde pública, em razão da sua alta prevalência e a possível progressão para a cirrose e/ou carcinoma hepatocelular (CASTRO PCS, et al., 2019).

A fisiopatologia apresenta-se com o excesso de gordura depositada nas células do fígado, que por sua vez induz o estresse oxidativo e a inflamação. Promove-se, portanto, um desequilíbrio metabólico. Esse processo perpassa pelo aumento da gliconeogênese e consequente diminuição da produção de glicogênio, sendo estes os principais fatores para a correlação da DHGNA à dislipidemia, bem como à RI. A doença não alcoólica, em sua forma mais grave, pode ocasionar a falência do órgão por intermédio da fibrose, ou seja, o fígado perde as suas funções e inúmeras manifestações sistêmicas são desencadeadas, a exemplo da hipertensão portal (OTT-FONTES PR, et al., 2020).

\section{Diagnóstico}

A partir da literatura consultada, observa-se que há uma avaliação inicial do acometimento hepático como parte do rastreamento de complicações do sobrepeso e obesidade. Em casos mais graves ou quando há dúvida quanto ao diagnóstico e tratamento, o médico hepatologista é incluído na avaliação e tratamento do caso. Porém, para o diagnóstico também é considerado a história clínica, sinais e sintomas e exames complementares (SIMONA L, et al., 2018).

Em relação a história clínica, para dizer que o paciente tem a doença, é necessário que a mesma tenha um histórico de consumo de álcool que não ultrapasse duas doses por dia para mulheres e três doses para os homens. Ou seja, uma dose de álcool corresponde a $30 \mathrm{ml}$ de bebida destilada ou $360 \mathrm{ml}$ de cerveja ou $120 \mathrm{ml}$ de vinho (SIMONA L, et al., 2018).

Já em relação aos sinais e sintomas, na maioria das vezes, é uma doença assintomática. Portanto, o diagnóstico é feito após o resultado de exames de imagem, principalmente ultrassonografia do abdômen. Exames de sangue e de imagem são solicitados para complementar a investigação da doença hepática (CASTRO PCS, et al., 2019).

Dos exames necessários, os principais são: (I) exames de sangue, visto que, as transaminases são os marcadores solicitados para auxiliar na avaliação do dano da gordura do fígado; (II) exames de imagem, sendo a ultrassonografia o método mais difundido, custo baixo e fácil quando se trata da DHGNA e a, (III) biópsia hepática, mas apenas em casos em que há dúvida do diagnóstico, por ser um exame invasivo e com riscos consideráveis (SIMONA L, et al., 2018).

\section{Epidemiologia}

A DHGNA se insere no campo da hepatologia, afetando-o mundialmente. Em paralelo ao aumento da disponibilidade de agentes antivirais eficazes e consequente redução do impacto das hepatites virais, a DHGNA tem se tornado rapidamente a causa mais comum de doença hepática crônica nos países ocidentais, tal qual o aumento significativo de enfermidades associadas à obesidade. Uma tendência semelhante é esperada nos países orientais nos próximos anos, com igual impacto dos fatores de risco do ocidente (SIMONA L, et al., 2018). 
Essa epidemia e suas consequências levaram especialistas de várias localidades a identificar estratégias eficazes para o diagnóstico, manejo e tratamento da DHGNA. As estimativas da prevalência de DHGNA variam de 6,3 a $33 \%$ em todo o mundo, com uma mediana de $20 \%$ na população em geral. A prevalência de EHNA na população geral dos EUA foi estimada em 2 a 12\% e a de NAFLD em 20 a $46 \%$. NAFLD é atualmente a terceira indicação mais frequente para o transplante de fígado, mas prevê-se que se torne a principal indicação em 2020, principalmente se associado ao aumento de enfermidades crônicas relacionadas às enfermidades progressivamente crônicas como obesidade e diabetes (ELIAS B, et al., 2018).

Em uma perspectiva mundial, houve um aumento da prevalência da DHGNA nos últimos 20 anos. Para além do padrão-ouro de diagnóstico (biópsia hepática), acredita-se que o advento de métodos de imagem menos invasivos e o aprimoramento de análises laboratoriais pode ter contribuído para a discrepância nos dados de prevalência de tal enfermidade nas últimas duas décadas (PERUMPAIL BJ, et al., 2017). Entretanto, a real prevalência da DHGNA é subestimada na população em geral, pois caráter predominantemente assintomático da doença repercute em sua descoberta acidental, devido a alterações em ultrassonografias e em enzimas de lesão hepática (ANDRADE GC, et al., 2016).

Segundo Perumpail BJ, et al. (2017), estima-se que os custos médicos e sociais referentes à DHGNA nos Estados Unidos consistam em US $\$ 292$ bilhões por ano. A projeção de custos relacionados aos cuidados de pacientes com DHGNA prevê que ocorra um aumento de $18 \%$ de 2000 a 2035 , e espera-se que a qualidade de vida dessas pessoas, no que tange à saúde, sofra um declínio.

\section{Fatores de Risco}

Dentro do que se pode considerar fatores de risco para DHGNA, podemos diferenciá-los em dois grandes distintos grupos: fatores de risco modificáveis e não modificáveis. A obesidade como comorbidade de base se torna uma porta significativa para o desenvolvimento de uma gama de fatores que podem culminar no desenvolvimento da DHGNA, à frente se pontuam esses fatores de forma sistemática para melhor compreensão (SIMONA L, et al., 2018)

A literatura recente indica um aumento da frequência da DHGNA associada à síndrome metabólica, evidenciando uma importante relação entre ambas e corroborando com a ideia de que a DHGNA pode consistir em uma manifestação hepática dessa síndrome (ANDRADE GC, et al., 2016).

É importante ressaltar que embora o conhecimento acerca da DHGNA se intensifique com o passar dos anos, várias dúvidas permanecem, inclusive sobre os mecanismos patogênicos e vias metabólicas influenciadas pelos fatores de risco. Assim, até que mais informações surjam e permitam desenvolver melhores estratégias diagnósticas e, é importante impulsionar a iniciativa global de diminuir a obesidade e aumentar a conscientização populacional acerca das doenças hepáticas relacionadas aos distúrbios metabólicos (YOUNOSSI ZM, 2018).

\section{Progressão da DHGNA e relação com a cirrose hepática}

A DHGNA apresenta várias fases de progressão, as quais incluem: esteatose hepática, EHNA, fibrose hepática, cirrose hepática, e pode, inclusive, evoluir para o carcinoma hepatocelular (KUDARAVALLI P e JOHN S, 2020). As condições compreendidas pelo termo DHGNA podem ser diagnosticadas por ultrassonografia, a partir da demonstração de esteatose hepática realizada pelo exame. Contudo, a análise histopatológica do tecido obtido por meio de biópsia hepática apresenta-se como padrão-ouro para o diagnóstico, permitindo classificar a atividade da doença, o estágio de fibrose hepática e a veracidade arquitetônica dos hepatócitos (HARDY T, et al., 2016).

A esteatose hepática configura-se como a principal marca histológica da DHGNA, evidenciada pela presença de gordura em mais de $5 \%$ dos hepatócitos em amostra proveniente de biópsia hepática, na ausência de inflamação, de fibrose e de consumo de mais de $30 \mathrm{~g}$ de álcool por dia (CASTRO L e SILVA G, 2015). Esse quadro consiste na forma exclusiva de apresentação de DHGNA pela maioria dos pacientes, configurando-se predominantemente assintomática. Quando apresenta sintomas previamente ao diagnóstico e tratamento, são normalmente inespecíficos e destacam-se fadiga, dor abdominal superior, distensão abdominal, sede e distúrbios do sono (KUDARAVALLI P e JOHN S, 2020). 
Estudos sugerem que pacientes com esteatose hepática apresentam menor chance de desenvolverem uma modalidade progressiva de DHGNA (HARDY T, et al., 2016). Segundo um estudo brasileiro que acompanhou a evolução de indivíduos acometidos por 10 anos, o risco de avanço da doença está diretamente relacionado à presença de EHNA na avaliação histológica. Ademais, em uma pesquisa distinta, é destacada a participação de outras comorbidades nessa progressão do quadro, tais quais dislipidemias, hipertensão, obesidade e diabetes mellitus tipo II, sendo a última referida como o maior fator metabólico atuante na progressão da DHGNA para cirrose hepática (CASTRO P, et al., 2019; LI B, et al., 2018).

A EHNA é caracterizada pela presença de padrão de esteatose hepática, que se associa à lesão hepática e à inflamação (com ou sem fibrose), na análise histológica (CASTRO L e SILVA G, 2015). Tal condição é dinâmica e está altamente associada à fibrose, de modo que pode regredir para a esteatose hepática, ou mesmo causar eventos fibróticos contínuos e culminar em cirrose hepática. A fibrose é esperada em pacientes com EHNA, visto que esses apresentam maior injúria hepática, e a remodelação tecidual, por sua vez, envolve eventos fibróticos. Por outro lado, as taxas de progressão da fibrose variam entre os indivíduos; em alguns casos, está rapidamente regride, ao passo que em outros evolui até mesmo após a resolução da EHNA (DIEHL AM e DAY C, 2017).

As doenças do fígado apresentam um denominador em comum, a cirrose, causada por fibrose, sendo a última crônica e persistente. Logo, o gerenciamento de risco deve ser prioridade no diagnóstico e/ou tratamento, uma vez que o objetivo é evitar a falência do órgão e, por consequência, disfunções sistêmicas que elevam o índice de morbimortalidade devido a formações nodulares difusas, bem como a desorganização vascular e lobular. A fibrose, no que tange à DHGNA, é gradual e cursa com algumas comorbidades consideradas mais frequentes na atualidade, a exemplo da síndrome metabólica. Assim sendo, estudos da história natural da evolução da fibrose em pacientes com DHGNA, alertam para a progressão da doença em um estágio a cada dez anos. No entanto, essa evolução pode variar entre os indivíduos, em virtude das singularidades do organismo de cada um (CASTRO PCS, et al., 2019).

A cirrose hepática representa o desfecho patológico da DHGNA, e será desenvolvida por aproximadamente $25 \%$ dos pacientes acometidos pela EHNA em algum momento de suas vidas, cerca de $2 \%$ dos adultos estadunidenses. A cirrose relacionada à EHNA caracteriza-se por fibrose extensa com formação de nódulo regenerativo, e é atualmente a segunda indicação mais comum para transplante de fígado nos Estados Unidos (CAUSSY C, et al., 2017; DIEHL AM e DAY C, 2017).

Além da relação do padrão histológico de injúria hepática com o desenvolvimento de cirrose, devido à maior probabilidade de pacientes com DHGNA desenvolverem o quadro, bem como da ligação da síndrome metabólica com tal afecção, estudos recentes têm determinado outros fatores de risco que aumentam as chances de progressão da DHGNA (CASTRO PCS, et al., 2019; HARDY T, et al., 2016; LI B, et al., 2018).

Acredita-se que polimorfismos genéticos podem proporcionar aumento de $30-50 \%$ do risco de desenvolvimento de cirrose hepática. Estudos de associação do genoma identificaram algumas variantes genéticas associadas à piora do prognóstico em pacientes com DHGNA, dentre as quais o polimorfismo de nucleotídeo único no gene PNPLA3 vem sendo fortemente associado ao desenvolvimento de cirrose hepática. Ademais, também há evidências de que pacientes com idade mais avançada apresentam maiores taxas de fibrose hepática risco mais significativo de evolução para cirrose. Todavia, ainda se busca compreender se a relação entre a idade e a progressão para cirrose realmente existe, ou o maior risco está ligado à duração da DHGNA (LI B, et al., 2018).

Outro fator contribuinte para o avanço da doença consiste no histórico familiar. De acordo com estudo de coorte realizado por Caussy C, et al. (2017), familiares de primeiro grau de pacientes com cirrose hepática relacionada à DHGNA têm 12 vezes maior risco de desenvolver fibrose avançada quando comparados a familiares de pacientes do grupo-controle, sugerindo que a cirrose hepática decorrente da DHGNA apresenta caráter hereditário.

O risco de morte aumenta substancialmente em pacientes com fibrose avançada, a qual aumenta a probabilidade de desenvolvimento de cirrose hepática e de câncer primário do fígado, sendo o mais frequente o Carcinoma Hepatocelular ( $\mathrm{CHC}$ ). A fisiopatologia da carcinogênese do CHC na DHGNA ainda não é clara, 
mas alguns estudos ratificam o papel da última como fator de risco para tal processo. Em consonância com um estudo realizado pela Sociedade Brasileira de Hepatologia, o $\mathrm{CHC}$ em pacientes com DHGNA está relacionado à EHNA no Brasil e também é observado em indivíduos não-cirróticos. Tendo esse cenário em vista, evidencia-se a necessidade de medidas preventivas, visto que o aumento do número de casos de DHGNA também influencia a prevalência de cirrose hepática e de carcinoma hepatocelular relacionados à EHNA (COTRIM HP, et al., 2016; DIEHL AM e DAY C, 2017; PERUMPAIL BJ, et al., 2017).

\section{Associação da doença a outras comorbidades}

De acordo com Vilar-Gomez E, et al. (2018), entre os pacientes com DHGNA que apresentam fibrose avançada, aqueles com cirrose correm um risco significativamente maior de descompensação hepática (70\%), carcinoma hepatocelular (17\%) e morte ou transplante do fígado em comparação com aqueles que apresentam fibrose em ponte, sendo que neste tipo de manifestação aumenta a predisposição a eventos vasculares e neoplasias malignas não hepáticas.

Ainda que na DHGNA o desenvolvimento de fibrose hepática seja maior devido, principalmente, à EHNA, alguns pacientes com outras comorbidades apresentam quadros de fibrose sem apresentar manifestações da EHNA. Nesse sentido, a RI parece ser um fator primordial para a DHGNA promover fibrose significativa em pacientes severamente obesos (PELUSI S, et al., 2019). Vale ressaltar que na síndrome metabólica, grupo de alterações metabólicas em que a obesidade visceral está inserida, a prevalência de fibrose grave, importante preditor de complicações hepáticas e extra-hepáticas da DHGNA está relacionada com a maior quantidade de eventos advindos desta síndrome (PETTA S, et al., 2017).

O Diabetes Mellitus Tipo 2 (DMT2) configura-se como uma comorbidade de alto impacto no prognóstico da DHGNA, sendo estimado que a prevalência global de DHGNA em pessoas com tal doença seja mais que o dobro em comparação à população geral, revelando também taxas significativas de EHNA e fibrose hepática avançada. Este cenário agrava-se proporcionalmente quando há associação positiva entre DMT2 e o aumento do IMC, especialmente na faixa entre 35-44,9kg/m² (JARVIS H, et al., 2020; OTT-FONTES PR, et al., 2020).

Importantes elementos da fisiopatologia da Doença Renal Crônica (DRC) como dislipidemia aterogênica, liberação de mediadores pró-inflamatórios, pró-coagulantes, pró-oxidantes e pró-fibrogênicos são predispostos pela DHGNA. Tal dinâmica acontece por meio da ativação da via de sinalização do fator nuclear kappa B (NF-kB), estimulando a transcrição de genes que estimulam a inflamação crônica sistêmica, resultando no aumento da produção de citocinas intra-hepáticas presente na DHGNA (TARGHER G e BYRNE CD, 2017).

Pacientes infectados pelo vírus da Imunodeficiência Humana (HIV) que não possuem outras causas de doença hepática, como hepatite viral ou abuso de álcool, apresentam anormalidades no que diz respeito ao funcionamento das enzimas hepáticas. Dessa forma, não só os fatores metabólicos são responsáveis pelo desenvolvimento de DHGNA em pacientes soropositivos, mas também tem sido apontado que a terapia antirretroviral pode comprometer os hepatócitos, seja por toxicidade mitocondrial, acidose láctica ou esteatose hepática (PIRES LB, et al., 2020).

\section{Tratamento}

A princípio, o tratamento da DHGNA tem como objetivo controlar os fatores de risco, prevenir a progressão da doença para formas mais graves e prevenir complicações. Portanto, mudanças no estilo de vida devem ser incentivadas pela equipe multidisciplinar por meio de atividade física regular e dietas equilibradas. Portanto, a atividade física reduz os adipócitos e aumenta a massa magra, contribuindo no controle do peso e reduzindo a glicemia, a hipertensão arterial e o risco cardiovascular (JARVIS H, et al., 2020; OTT-FONTES PR, et al., 2020).

No que diz respeito à dieta, tem sido visto que a ingestão de carboidratos complexos, o aumento do consumo de frutose, gorduras saturadas e produtos industrializados, em conjunto com o sedentarismo, favorece a desregulação metabólica, acarretando em quadros de obesidade e DHGNA. Nesse sentido, ácidos graxos monoinsaturados, encontrados no azeite de oliva, oleaginosas e abacate são benéficos para pacientes com DHGNA, já que incentiva a redução dos níveis de colesterol e triglicerídeos intra-hepáticos (DUARTE SM, et al., 2019). 
Como alternativa terapêutica, o GS-0976 demonstra relevância no tratamento da EHNA por ser um inibidor alostérico da acetil-CoA-carboxilase 1 (ACC1) e acetil-CoA-carboxilase 2 (ACC2), enzimas responsáveis respectivamente por catalisar a formação de malonil-CoA, substrato essencial para a biossíntese de ácidos graxos no citosol, e inibir alostericamente a carnitina palmitoil-transferase 1 (CPT1), a qual transporta ácidos graxos nas mitocôndrias para a beta-oxidação. Dessa forma, o bloqueio enzimático feito pela pequena molécula em questão diminuiu a esteatose hepática, inflamação e a $\mathrm{Rl}$, garantindo também redução nos marcadores de fibrose hepática (LOOMBA R, et al., 2018).

\section{CONSIDERAÇÕES FINAIS}

Por meio da análise bibliográfica deste estudo, evidencia-se que a DHGNA consiste no acúmulo de gordura nos hepatócitos de indivíduos que não consomem ou consomem menos que $30 \mathrm{~g}$ de álcool por dia, propiciando um distúrbio metabólico. Apesar de ser um transtorno hepático prevalente no mundo, a DHGNA apresenta uma evolução progressiva e geralmente assintomática, resultando em diagnósticos tardios, o que justifica o risco de evoluir para cirrose e/ou carcinoma hepatocelular e a necessidade de transplante hepático. Além disso, foi observado que a DHGNA está associada à síndrome metabólica, o que é preocupante, devido à elevada incidência de obesidade atualmente. Dessa forma, a biópsia do fígado é considerada padrão-ouro para avaliar o estágio histopatológico em que o paciente se encontra.

\section{REFERÊNCIAS}

1. ANDRADE GC, et al. Non-alcoholic fatty liver disease (NAFLD) in different populations: A clinical and epidemiological study - sample of São José do Rio Preto. Revista da Associação Médica Brasileira, 2016; 62(3): 218-226.

2. CABALLERÍA L, et al. High Prevalence of Liver Fibrosis Among European Adults With Unknown Liver Disease: A Population-Based Study. Clinical Gastroenterology and Hepatology, 2018; 16: 1138-1145.

3. CASTERA L. Diagnosis of non-alcoholic fatty liver disease/non-alcoholic steatohepatitis: non-invasive tests are enough. Liver International, 2018; 38: 67-70.

4. CASTRO L, SILVA G. Hígado Graso no Alcohólico. Revista Médica Clínica Las Condes, 2015; 26(5): 600-612.

5. CASTRO PCS, et al. Evaluation of Progression of Hepatic Fibrosis in a Group of Patients with Non-Alcoholic Fatty Liver Disease Accompanied for 10 years. Arq. Gastroenterol, 2019; 53(3): 256- 260.

6. CAUSSY C, et al. Nonalcoholic fatty liver disease with cirrhosis increases familial risk for advanced fibrosis. The Journal of clinical investigation, 2017; 127(7): 2697-2704.

7. COTRIM H, et al. Nonalcoholic steatohepatitis and hepatocellular carcinoma: Brazilian survey. Clinics, 2016; 71(5): $281-284$.

8. DIEHL AM, DAY C. Cause, pathogenesis, and treatment of nonalcoholic steatohepatitis. New England Journal of medicine, 2017 ; 377: 2063-2072.

9. DUARTE SM, et al. Impact of current diet at the risk of non-alcoholic fatty liver disease (NAFLD). Arquivos de Gastroenterologia, 2019; 56(4): 431-439.

10. ELIAS B, et al. An Integrated Understanding of the Rapid Metabolic Benefits of a Carbohydrate-Restricted Diet on Hepatic Steatosis in Humans, clinical and translational report. Cell Metabolism, 2018; 27: 559-571.

11. HARDY T, et al. Nonalcoholic Fatty Liver Disease: Pathogenesis and Disease Spectrum. Annual review of pathology, $2016 ; 11: 451$ 496.

12. JARVIS $\mathrm{H}$, et al. Metabolic risk factors and incident advanced liver disease in non-alcoholic fatty liver disease (NAFLD): A systematic review and meta-analysis of population-based observational studies. PLoS medicine, 2020; 17(4): 1-20.

13. KUDARAVALLI P, JOHN S. Nonalcoholic Fatty Liver. Florida: StatPearls Publishing, 2020; 19p.

14. LI B, et al. Nonalcoholic Fatty Liver Disease Cirrhosis: A Review of Its Epidemiology, Risk Factors, Clinical Presentation, Diagnosis, Management, and Prognosis. Canadian Journal of Gastroenterology and Hepatology, 2018; 2(07).

15. LOOMBA R, et al. GS-0976 reduces hepatic steatosis and fibrosis markers in patients with nonalcoholic fatty liver disease. Gastroenterology, 2018; 155(5): 1463-1473.

16. MATOS LC. Fígado Gordo Não Alcoólico: Revisão da Literatura. Gazeta médica, 2017; 4(1): 43-49.

17. OTT-FONTES PR, et al. Comparação da gravidade da doença hepática gordurosa não alcoólica de pacientes obesos diabéticos e não diabéticos. Revista do Colégio Brasileiro de Cirurgiões, 2020; 47: 1-8.

18. PARENTE DB. Métodos de imagem na avaliação da doença hepática gordurosa não alcoólica. Colégio Brasileiro de Radiologia, 2020; 53(2): 9-10.

19. PELUSI S, et al. Prevalence and risk factors of significant fibrosis in patients with nonalcoholic fatty liver without steatohepatitis. Clinical Gastroenterology and Hepatology, 2019; 17(11): 2310-2319.

20. PERUMPAIL BJ, et al. Clinical epidemiology and disease burden of nonalcoholic fatty liver disease. World journal of gastroenterology, 2017; 23(12): 8263-8276.

21. PETTA S, et al. Metabolic syndrome and severity of fibrosis in nonalcoholic fatty liver disease: An age-dependent risk profiling study. Liver International, 2017; 37(9): 1389-1396.

22. PIRES LB, et al. Non-alcoholic fatty liver disease in patients infected with human immunodeficiency virus: a systematic review. Revista da Associação Médica Brasileira, 2020; 66(1): 81-86.

23. SIMONA L, et al. Current guidelines for the management of non-alcoholic fatty liver disease: A systematic review with comparative analysis. World Journal of Gastroenterology, 2018; 24(30): 3361-3373.

24. TARGHER G, BYRNE CD. Non-alcoholic fatty liver disease: an emerging driving force in chronic kidney disease. Nature Reviews Nephrology, 2017; 13(5): 297-310.

25. VILAR-GOMEZ E, et al. Fibrosis severity as a determinant of cause-specific mortality in patients with advanced nonalcoholic fatty liver disease: a multi-national cohort study. Gastroenterology, 2018; 155(2): 443-457.

26. YOUNOSSI ZM. Non-alcoholic fatty liver disease - A global public health perspective. Journal of Hepatology, 2018; 70(3): 531-544. 